\title{
Follicular Transplantation, Microneedling, and Adjuvant Narrow-band Ultraviolet-B Irradiation as Cost-Effective Regimens for Palmar-Plantar Vitiligo: A Pilot Study
}

Amir Feily ${ }^{1}$, Abdollah Firoozifard ${ }^{1}$, Toktam Sokhandani ${ }^{1}$, Perla Elosegui-Rodriguez ${ }^{2}$, Evian Perez-Rivera ${ }^{2}$, Christopher S. Lange ${ }^{3}$, Masoomeh Hosseinpoor ${ }^{4}$, Marigdalia K. Ramirez-Fort ${ }^{5}$

1. Dermatology, Jahrom University of Medical Sciences, Jahrom, IRN 2. Pathology, San Juan Bautista School of Medicine, Caguas, PRI 3. Radiation Oncology, State University of New York Downstate Health Sciences University, Brooklyn, USA 4. Otolaryngology, Mashhad University of Medical Sciences, Mashhad, IRN 5. Life Sciences, Biofort Corp., Guaynabo, PRI

Corresponding author: Marigdalia K. Ramirez-Fort, marigdalia@biofort.io

\section{Abstract}

Treatment of refractory palmar-plantar vitiligo is particularly challenging because the skin in these regions has a limited supply of follicle-derived melanocytic stem cells. Autologous hair transplantation monotherapy is effective in some forms of vitiligo through the provision of melanocytic stem cells. $\mathrm{CO}_{2}$ laser followed by exposure to light (i.e., sunlight or narrow-band ultraviolet-B [nbUVB]) has independently shown to be an effective treatment strategy. Recently, it was found that the combination of hair transplantation and $\mathrm{CO}_{2}$ laser followed by nbUVB exposure had superior efficacy to either modality as monotherapy. Similar to $\mathrm{CO}_{2}$ laser, microneedling produces skin cell proliferation and releases propigmentary cytokines. Given the important role of the cytokines in vitiliginous skin, microneedling may also be an effective therapeutic modality for refractory vitiligo. Herein, we conducted a pilot study to evaluate the efficacy of hair transplantation and $\mathrm{CO}_{2}$ laser or microneedling followed by nbUVB. Microneedling and fractional $\mathrm{CO}_{2}$ laser in combination with hair transplantation and nbUVB both demonstrated utility in the induction of repigmentation in refractory palmar-plantar vitiligo; however, a larger trial would be needed to determine a difference in treatment efficacy. Nonetheless, microneedling is cost-effective and requires minimal training; therefore, microneedling can be easily incorporated into standard dermatological practice.

Received 03/13/2020

Review began 04/05/2020 Review ended 04/19/2020 Published 04/28/2020

\section{() Copyright 2020}

Feily et al. This is an open access article distributed under the terms of the Creative Commons Attribution License CC-BY 4.0., which permits unrestricted use, distribution, and reproduction in any medium, provided the original author and source are credited.
Categories: Dermatology, Radiation Oncology, Allergy/Immunology

Keywords: vitiligo, micro needling, nbuvb, melanocytic stem cells, hair transplantation, co2 fractional laser, ultraviolet radiation (uvr) therapy

\section{Introduction}

Treatment of stable and refractory palmar-plantar vitiligo is particularly challenging because the skin in these regions is inherently void of hair follicles. Follicles are an important source of melanocytic stem cells needed for repigmentation [1-3]. Therefore, hair follicle transplantation, while time-consuming, is a valuable mechanism to introduce pigmentary stem cells into the palmar-plantar regions [4-7]. An increasing amount of literature underlines the efficacy of hair follicle transplantation as a valid monotherapy for cutaneous repigmentation [4-7]. Currently, hair follicle transplantation is being used in different therapeutic protocols, which combine fractionated $\mathrm{CO}_{2}$ laser or microneedling with narrow-band ultraviolet-B (nbUVB) phototherapy and topical steroids [8-12]. Both fractional $\mathrm{CO}_{2}$ laser and microneedling as therapeutic options for vitiligo seem to be independently useful, but there are no comparative studies to date.

In the recent past, our group described the validity and efficacy of a quadrimodal therapy with hair transplantation, fractionated $\mathrm{CO}_{2}$ laser, topical steroids, and nbUVB for refractory vitiligo [9]. The improved efficacy of the quadrimodal treatment occurred by the presumed mechanisms of melanotoxin depletion, increased topical steroid delivery through ablative channels, and induced upregulation of pro-pigmentary cytokines within the lesional microenvironment; collectively, these changes combined with nbUVB stimulation are thought to enhance stem cell viability and melanin production [8-12]. Although nbUVB can be quite effective as monotherapy, the lack of hair follicles (and source of melanocytic stem cells) in palmarplantar vitiliginous skin limits its use in this vitiligo phenotype.

Similar to fractionated $\mathrm{CO}_{2}$ laser, microneedling is a therapeutic modality that produces skin cell proliferation and releases pro-pigmentary cytokines [12,13]. Specifically, it accomplishes this by inserting needles onto the skin and promoting healing [14]. Given the important role of cytokines in vitiliginous skin, microneedling may be a cost-effective therapeutic modality (as compared to fractional $\mathrm{CO}_{2}$ laser) for 
refractory vitiligo $[8,9,12,13,15]$. The traumatic inflammatory infiltrate from microneedle punctures may optimize regional antigen presentation and wound healing, leading to the removal of pathogenic cells and melanotoxins.

On the other hand, fractional $\mathrm{CO}_{2}$ laser is a tissue-selective treatment used for many dermatological diseases that emits light energy and fractionates it into microbeams that are delivered in a certain number of sessions, or fractions, to the tissue [16]. The microbeams are not pigment-selective and are absorbed by water, mostly found in soft tissues. The energy is delivered at high peaks and short duration to induce inflammation in the intended tissue and minimizes damage of the normal surrounding tissue. The devices that emit the fractional $\mathrm{CO}_{2}$ laser cost approximately $\$ 25,000$, whereas the microneedling devices cost approximately $\$ 1,000$, but prices vary by vendor. Compared with fractional CO2 laser, the cost of the microneedling procedure is significantly less to the practicing dermatologist; therefore, microneedling quadrimodality (i.e., microneedling, hair transplantation, topical steroids, nbUVB) is a protocol that could be conveniently incorporated into standard practice for the therapeutic management of refractory palmarplantar vitiligo.

IIn this study, we aim to compare the efficacy of microneedling with fractionated $\mathrm{CO}_{2}$ laser, as part of a multimodal regimen including hair follicle transplantation, nbUVB, and topical clobetasol solution, in the treatment of stable and refractory palmar-plantar vitiligo.

\section{Materials And Methods}

A prospective pilot study was conducted from January 2015 to January 2016. Twenty patients (10 women; 10 men) with Fitzpatrick skin type IV were enrolled in the study; two male patients withdrew consent prior to starting treatment. All patients were diagnosed with stable and refractory palmar-plantar vitiligo by boardcertified dermatologists. Stable and refractory vitiligo was defined as lesions that did not progress over a $12-$ month timeframe and that had not previously responded to standard monotherapies or combined ones (e.g. topical steroids, nbUVB) (Table 1). Major exclusionary criteria included (1) hypersensitivity to laser and hair transplantation materials, (2) a history of photosensitivity, (3) a personal history of keloid formation, and (4) a personal history of Koebnerization.

\begin{tabular}{|c|c|c|}
\hline Treatment & Duration & Number of patients \\
\hline Topical corticosteroids & 6 weeks & 15 \\
\hline Topical calcineurin Inhibitors & 2 months & 5 \\
\hline Nb-UVB & 15 weeks & 20 \\
\hline Nb-UVB + topical corticosteroids & 15 weeks & 5 \\
\hline
\end{tabular}

TABLE 1: Prior failed treatment modalities in enrolled patients.

$\mathrm{Nb}$, narrow-band; UVB, ultraviolet $\mathrm{B}$ rays

Comparable vitiliginous lesions were selected from both sides of the body. On day 0 , heavily pigmented follicular grafts were harvested from the scalp by follicular unit extraction and transplanted in a 1-cm grid pattern throughout the selected lesions. At days $30 \pm 4$ and $60 \pm 4$, left-sided lesions received single fractions with a fractional CO2 laser, MX-7000 (10,600 nm, 100-MJ pulse energy, and 200 spots/cm3 in static mode) (Daeshin Enterprise Corporation, IDS CO, Seoul, Korea), under topical anesthesia with lidocaine-prilocaine cream applied for 20 minutes prior to intervention. Right-sided lesions received 1.5- to 2-mm needle lengthassisted microneedling until pinpoint bleeding was achieved. Topical anesthesia with lidocaine-prilocaine cream was used for 20 minutes prior to microneedling. On days $30 \pm 4$ and $60 \pm 4$ after primary investigational treatment (i.e., fractional CO2 laser or microneedling), silver sulfadiazine ointment was applied to the treated skin, twice daily, for five days without occlusion.

On day $41 \pm 2$, right- and left-sided lesional skin received nbUVB phototherapy (Dermalight 1,000/1,000 L, $800 \mathrm{~W}$; power supply: $230 \mathrm{~V} / 50 \mathrm{~Hz}$; Dr. Honle Medizinitechnik GmbH, Gilching, Germany) three times a week until day $60 \pm 4$; phototherapy was restarted on day $71 \pm 2$. The phototherapeutic dose was increased by $15 \%$ at each treatment interval and was completed by day $100 \pm 4$ (for a total of 12 weeks of treatment). Clobetasol $45 \%$ solution ( $45 \%$ clobetasol in 100-mL isopropyl alcohol) was applied twice daily throughout the phototherapy phase of the study.

The diameter of repigmentation around each graft was photographed and measured with appropriately calibrated calipers at baseline: days $30 \pm 4,60 \pm 4$, and $100 \pm 4$. Treatment toxicities were evaluated at each 


\section{Cureus}

patient visit and measured according to the Common Terminology Criteria for Adverse Events (CTCAE v4.0). On day $100 \pm 4$, patients completed a treatment satisfaction questionnaire (scale of 0 to 10 , unsatisfied to highly satisfied).

Data were analyzed by the SPSS software (IBM Corp., Armonk, NY, USA) using the Mann-Whitney and Friedman tests.

\section{Results}

Twenty patients (10 women; 10 men) with Fitzpatrick skin type IV were enrolled in the study; two male patients withdrew consent prior to starting treatment. The mean patient age was 30.22 years. The mean duration of disease \pm SD was $10.4 \pm 5$.8 years. There was an even distribution (i.e., $1: 1$ ratio) of palmar and plantar lesions treated.

A total of 95\% of transplanted follicles survived; all remaining follicles maintained pigment throughout the study. At days $30 \pm 4$ and $60 \pm 4$, there was $0.21 \mathrm{~mm}$ and $0.25 \mathrm{~mm}$ of repigmentation after hypofractionated $\mathrm{CO}_{2}$ laser compared with $0.08 \mathrm{~mm}$ and $0.17 \mathrm{~mm}$ in lesions treated with microneedling $(\mathrm{p}=0.74$ and $\mathrm{p}=0.86$ for days $30 \pm 4$ and $60 \pm 4$, respectively) (Figure $1 A-C$ ) (Table 2). By day $100 \pm 4,78 \%$ of grafts demonstrated repigmentation, which measured $0.25 \mathrm{~mm}$ after hypofractionated $\mathrm{CO}_{2}$ laser and $0.25 \mathrm{~mm}$ after

microneedling $(p=0.84)$. By power analysis, 93 patients are needed to have an $80 \%$ chance of not rejecting a true difference in efficacy between hypofractionated $\mathrm{CO}_{2}$ laser and microneedling.

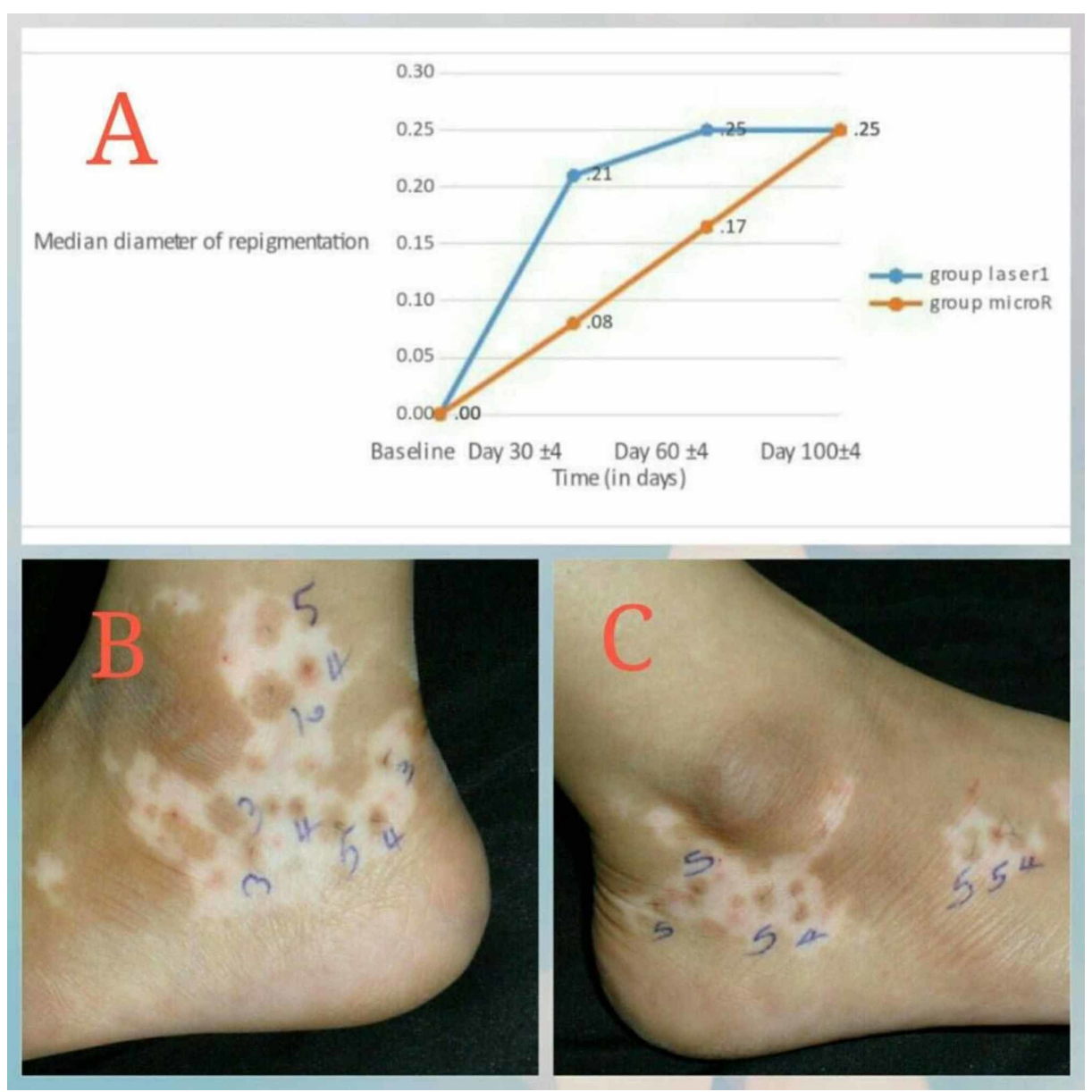

FIGURE 1: Rate of repigmentation by treatment arms.

(A) Graphically expressed diameter of repigmentation around each graft. (B and C) Photographed repigmentation. 


\section{Cureus}

\begin{tabular}{|c|c|c|c|}
\hline \multirow{2}{*}{ Follow-up time } & \multicolumn{2}{|l|}{ Treatment arms } & \multirow{2}{*}{ p-Value } \\
\hline & Laser $(\mathrm{mm})$, median (range) & Microneedling (mm), median (range) & \\
\hline Baseline & $0.00(0-0)$ & $0.00(0-0)$ & - \\
\hline Day $30 \pm 4$ & $0.21(0-2)$ & $0.08(0-1)$ & 0.74 \\
\hline Day 60ะ4 & $0.25(0-2.57)$ & $0.17(0-1.30)$ & 0.86 \\
\hline Day $100 \pm 4$ & $0.25(0-3)$ & $0.25(0-1.66)$ & 0.84 \\
\hline
\end{tabular}

TABLE 2: Diameter of repigmentation by treatment arms.

All patients experienced grade 1 erythema and grade 1 pain in both treatment arms after fractional $\mathrm{CO}_{2}$ laser and microneedling. Grades 2 to 5 toxicities were not identified. High patient satisfaction was noted in both treatment groups: a treatment satisfaction questionnaire (i.e., patient-reported outcome forms) did not identify a difference in patient preference toward one of the two modalities.

\section{Discussion}

Our pilot study indicates that quadrimodal therapy with microneedling or hypofractionated $\mathrm{CO}_{2}$ laser is efficacious in stable and refractory palmar-plantar vitiligo. The $78 \%$ overall response rate achieved by our patients supports that both microneedling and hypofractionated $\mathrm{CO}_{2}$ laser have similar efficacies in improving pigmentation of vitiliginous lesions when follicular transplantation, topical steroids, and nbUVB are incorporated $[1,17]$. Furthermore, both modalities result in comparable patient satisfaction.

The initial slope of the hypofractionated $\mathrm{CO}_{2}$ laser repigmentation curve was steeper when compared with microneedling, but the difference was not significant (Figure 1). The faster rate of repigmentation might be explained by the added benefit of the ablative channels created by the $\mathrm{CO}_{2}$ laser. Our pilot study was not powered to detect non-inferiority. A larger trial of at least 93 patients is needed to evaluate non-inferiority of either modality, but costs versus benefits support the use of microneedling in practices that do not have readily accessible fractional $\mathrm{CO}_{2}$ laser capabilities.

Prior studies have evaluated the efficacy of fractional $\mathrm{CO}_{2}$ laser (without transplantation of melanocytic stem cells) in combination with phototherapy (e.g., sunlight or targeted UVB) $[10,18]$. The results of these studies are contradictory. Fractional $\mathrm{CO}_{2}$ laser followed by sunlight (i.e., wide range of UV wavelengths) exposure resulted in acceptable repigmentation of refractory vitiligo, with a repigmentation rate ranging from $24 \%$ to $74 \%$ [10]. However, fractional $\mathrm{CO}_{2}$ laser followed by targeted UVB with topical steroids had no improvement in pigmentation compared with targeted UVB and topical steroids alone [18]. The latter findings suggest that the addition of $\mathrm{CO}_{2}$ laser treatment alone does not improve the efficacy of UV light with topical steroids. $\mathrm{CO}_{2}$ laser hypofractionation and transplantation of viable melanocytic stem cells is required to improve repigmentation [9]. We optimized the propigmentary environment by hypofractionating the $\mathrm{CO}_{2}$ laser sessions from 10 sessions to 2 sessions to prevent ablation of viable, residing

melanocytes $[9,18]$. We also implanted richly pigmented hair follicles as a source of healthy melanocytic stem cells [9]. $\mathrm{CO}_{2}$ laser hypofractionation should theoretically minimize the risk of vitiliginous Koebnerization, thereby leading to better clinical results $[9,18]$.

Stanimirovic et al. compared latanoprost in combination with nbUVB phototherapy with latanoprost plus one session of $0.5-\mathrm{mm}$ needle length-assisted skin microneedling in combination with nbUVB phototherapy to induce repigmentation in refractory vitiligo lesions [19]. The results showed that latanoprost with nbUVB was effective, but microneedling had no improvement upon this efficacy. The effectiveness of microneedling observed in our study may be explained by differences in session numbers, technique, and medical equipment. The performance of more microneedling sessions with longer needle lengths (i.e., Dermaroller ${ }^{\circledR}$ versus Dermapen ${ }^{\circledR}$ ) should induce a larger inflammatory infiltrate and propigmentary cytokine release.

Hair follicle transplantation was standard to both arms of the study. A potential adverse outcome with the use of melanocytic stem cells from hair follicles is the poor cosmesis of new hairs on the palmar and plantar skin. Therefore, patient education should be provided prior to the hair follicle transplantation procedure. In our experience, five patients showed hair growth in the treated areas, which was resolved with laser hair removal performed after complete patch repigmentation. 


\section{Conclusions}

Microneedling or fractional $\mathrm{CO}_{2}$ laser in combination with hair transplantation, topical steroids, and nbUVB are utile and effective in the induction of repigmentation in stable and refractory palmar-plantar vitiligo. The initial rate of repigmentation with the hypofractionated doses of fractional $\mathrm{CO}_{2}$ laser was faster than with microneedling, but this difference was not significant with the sample size of our pilot study. A larger trial would be needed to determine a difference in treatment efficacy. However, microneedling is more costeffective and requires minimal training; therefore, microneedling can be easily and efficiently incorporated into standard dermatological practice.

\section{Additional Information \\ Disclosures}

Human subjects: Consent was obtained by all participants in this study. Jahrom University of Medical Sciences Ethics Committee issued approval IR.JUMS.REC.1394.184. Iranian Registry of Clinical Trials number: IRCT2017032530808N3. Animal subjects: All authors have confirmed that this study did not involve animal subjects or tissue. Conflicts of interest: In compliance with the ICMJE uniform disclosure form, all authors declare the following: Payment/services info: All authors have declared that no financial support was received from any organization for the submitted work. Financial relationships: All authors have declared that they have no financial relationships at present or within the previous three years with any organizations that might have an interest in the submitted work. Intellectual property info: Ultraviolet radiation (UVR) therapy in treating human disease. Other relationships: All authors have declared that there are no other relationships or activities that could appear to have influenced the submitted work.

\section{References}

1. Arrunategui A, Arroyo C, Garcia L, Covelli C, Escobar C, Carrascal E, Falabella R: Melanocyte reservoir in vitiligo. Int J Dermatol. 1994, 33:484-487. 10.1111/j.1365-4362.1994.tb02860.x

2. Czajkowski R: Comparison of melanocytes transplantation methods for the treatment of vitiligo . Dermatol Surg. 2004, 30:1400-1405. 10.1111/j.1524-4725.2004.30435.x

3. Czajkowski R, Placek W, Drewa T, Kowaliszyn B, Sir J, Weiss W: Autologous cultured melanocytes in vitiligo treatment. Dermatol Surg. 2007, 33:1027-1036. 10.1111/j.1524-4725.2007.33216.x

4. Babu A, Thappa DM, Jaisankar TJ: Punch grafting versus suction blister epidermal grafting in the treatment of stable lip vitiligo. Dermatol Surg. 2008, 34:166-178. 10.1111/j.1524-4725.2007.34034.x

5. Li J, Fu WW, Zheng ZZ, Zhang QQ, Xu Y, Fang L: Suction blister epidermal grafting using a modified suction method in the treatment of stable vitiligo: a retrospective study. Dermatol Surg. 2011, 37:999-1006. 10.1111/j.1524-4725.2011.01966.x

6. Mapar MA, Safarpour M, Mapar M, Haghighizadeh MH: A comparative study of the mini-punch grafting and hair follicle transplantation in the treatment of refractory and stable vitiligo. J Am Acad Dermatol. 2014, 70:743-747. 10.1016/j.jaad.2013.11.044

7. Na GY, Seo SK, Choi SK: Single hair grafting for the treatment of vitiligo . J Am Acad Dermatol. 1998, 38:580584. 10.1016/s0190-9622(98)70121-5

8. El Mofty M, Esmat S, Hunter N, Mashaly HM, Dorgham D, Shaker O, Ibrahim S: Effect of different types of therapeutic trauma on vitiligo lesions. Dermatol Ther. 2017, 30:5. 10.1111/dth.12447

9. Feily A, Seifi V, Ramirez-Fort MK: Fractional CO2 laser pretreatment to autologous hair transplantation and phototherapy improves perifollicular repigmentation in refractory vitiligo: a randomized, prospective, halflesion, comparative study. Dermatol Surg. 2016, 42:1082-1088. 10.1097/DSS.0000000000000844

10. Helou J, Maatouk I, Obeid G, Moutran R, Stephan F, Tomb R: Fractional laser for vitiligo treated by 10,600 nm ablative fractional carbon dioxide laser followed by sun exposure. Lasers Surg Med. 2014, 46:443-448. 10.1002/1sm.22260

11. Mehraban S, Feily A: 308nm excimer laser in dermatology . J Lasers Med Sci. 2014, 5:8-12.

12. Seif El Nasr H, Shaker OG, Fawzi MM, El-Hanafi G: Basic fibroblast growth factor and tumour necrosis factor alpha in vitiligo and other hypopigmented disorders: suggestive possible therapeutic targets. J Eur Acad Dermatol Venereol. 2013, 27:103-108. 10.1111/j.1468-3083.2011.04368.x

13. Liebl H, Kloth LC: Skin cell proliferation stimulated by microneedles. J Am Coll Clin Wound Spec. 2012, 4:26. Accessed: April 25, 2020: 10.1016/j.jccw.2012.11.001

14. Iriarte C, Awosika O, Rengifo-Pardo M, Ehrlich A: Review of applications of microneedling in dermatology . Clin Cosmet Investig Dermatol. 2017, 10:289-298. Accessed: April 25, 2020: 10.2147/CCID.S142450

15. Lotti T, Hercogova J, Fabrizi G: Advances in the treatment options for vitiligo: activated low-dose cytokines-based therapy. Expert Opin Pharmacother. 2015, 16:2485-2496. 10.1517/14656566.2015.1087508

16. Omi T, Numano K: The role of the CO2 laser and fractional CO2 laser in dermatology . Laser Ther. 2014, 23:49-60. 10.5978/islsm.14-RE-01

17. Agrawal K, Agrawal A: Vitiligo: surgical repigmentation of leukotrichia. Dermatol Surg. 1995, 21:711-715. 10.1111/j.1524-4725.1995.tb00275.x

18. Kanokrungsee S, Chanprapaph K, Chaiyabutr C, Vachiramon V: A comparative study of combined treatment with fractional carbon dioxide and targeted ultraviolet B phototherapy for facial vitiligo. Lasers Med Sci. 2016, 31:1343-1349. 10.1007/s10103-016-1982-Z

19. Stanimirovic A, Kovacevic M, Korobko I, Situm M, Lotti T: Combined therapy for resistant vitiligo lesions: NB-UVB, microneedling, and topical latanoprost, showed no enhanced efficacy compared to topical latanoprost and NB-UVB. Dermatol Ther. 2016, 29:312-316. 10.1111/dth.12363 